\title{
Etiology of burning mouth syndrome: A review and update
}

\author{
Mari-Paz Minguez-Sanz ${ }^{1}$, Cesar Salort-Llorca ${ }^{2}$, Francisco-Javier Silvestre-Donat ${ }^{1,3}$ \\ ${ }^{1}$ Dental surgeon. Stomatology Unit. Doctor Peset University Hospital. Valencia (Spain) \\ ${ }^{2}$ Pharmacist. Specialist in Hospital Pharmacy \\ ${ }^{3}$ Assistant Professor. Department of Stomatology. Valencia University Medical and Dental School (Spain)
}

Correspondence:

Unidad de Estomatología

Hospital Universitario Dr. Peset (Consultas externas)

Juan de Garay s/ $n$

46017 - Valencia (Spain)

francisco.silvestre@uv.es

Received:24/02/2010

Accepted: $11 / 03 / 2010$

\author{
Minguez-Sanz MP, Salort-Llorca C, Silvestre-Donat FJ. Etiology of bur- \\ ning mouth syndrome: A review and update. Med Oral Patol Oral Cir \\ Bucal. 2011 Mar 1;16 (2):e144-8. \\ http://www.medicinaoral.com/medoralfree 01/v16i2/medoralv16i2p144.pdf \\ Article Number: $16865 \quad$ http://www.medicinaoral.com \\ (C) Medicina Oral S. L. C.I.F. B 96689336 - pISSN 1698-4447 - eISSN: 1698-6946 \\ eMail: medicina@medicinaoral.com \\ Indexed in: \\ Science Citation Index Expanded \\ Journal Citation Reports \\ Index Medicus, MEDLINE, PubMed \\ Scopus, Embase and Emcare \\ Indice Médico Español
}

\begin{abstract}
Introduction: Burning mouth syndrome (BMS) is characterized by an oral burning sensation in the absence of any organic disorders of the oral cavity. Although the cause of BMS is not known, a complex association of biological and psychological factors has been identified, suggesting the existence of a multifactorial etiology.

Material and Method: A PubMed/Medline search was used to identify articles describing the different hypotheses regarding the etiology of BMS, as well as the psychological and anatomical data upon which such hypotheses are fundamented.

Results: A review and update was made of the different hypotheses relating to the etiology of BMS (psychogenic factors, hormone disorders, neuropathic alterations, oral phantom pain, neuroplasticity and neuroinflammation), with a view to globally establishing possible relationships among them.

Conclusions: In-depth investigation is needed to clarify the etiopathogenic mechanisms of BMS and its triggering factors, in order to develop effective and individualized management strategies that can be extended to patients in each different BMS subgroup.
\end{abstract}

Key words: Oral phantom pain, etiology, psychogenic factors, neuroinflammatory model, neuroplasticity, burning mouth syndrome, neuropathic theory, hormone disorders.

\section{Introduction}

Burning mouth syndrome (BMS) is characterized by an oral burning sensation in the absence of any organic disorders of the oral cavity. Scala et al. (1) proposed a series of differential diagnostic criteria for identifying BMS. In this context they established a distinction between fundamental and additional criteria for diagnosing the disease (Table 1).
Although different etiological classifications of BMS have been developed, the true cause underlying the syndrome and which may fully explain the disease process has not been clearly established. As a result, it is difficult to find a classification capable of including all possible presentations of BMS.

A number of hypotheses have been proposed to explain the etiopathogenic mechanism of BMS. The 
Table 1. Criteria developed by Scala for the diagnosis of burning mouth syndrome.

\begin{tabular}{|ll|}
\hline \multicolumn{2}{|c|}{ FUNDAMENTAL INCLUSION CRITERIA: } \\
\hline 1. & Daily and deep burning sensation of the oral mucosa (bilateral) \\
\hline 2. & Burning sensation for at least 4-6 months \\
\hline 3. & Constant intensity, or increasing intensity during the day \\
\hline 4. & No worsening on eating or drinking. The symptoms may improve \\
\hline 5. & No interference with sleep \\
\hline & \\
\hline & ADDITIONAL INCLUSION CRITERIA: \\
\hline 6. & Dysgeusia and/or xerostomia \\
\hline 7. & Sensory or chemosensory alterations \\
\hline 8. & Mood changes or psychopathological alterations \\
\hline
\end{tabular}

first studies attributed great importance to endocrine alterations and tissue degenerative phenomena inherent to aging. Posteriorly, research into the etiology of BMS has explored neuropathic disorders as well as psychopathological factors, due to the high incidence of the latter in patients with BMS. In this context, a complex association of biological and psychological factors has been identified, suggesting the existence of a multifactorial etiology (2). Debate on the role of these factors in the etiology of BMS has led to many hypotheses, though no single theory has received unanimous acceptance to date.

The present study offers a review and update of the different hypotheses relating to the etiology of BMS, with a view to globally establishing possible relationships among them.

\section{Search Method}

A PubMed/Medline search was used to identify articles describing the different hypotheses regarding the etiology of BMS, as well as the psychological and anatomical data upon which such hypotheses are fundamented.

\section{Results}

The present review has been made considering the three basic references in the etiology of BMS identified to date: psychological disorders, hormone alterations and disorders of the nervous system.

1.- Psychological disorders such as depression and anxiety play an important role in the modulation of pain perception, being able to increase or decrease nerve transmission from the peripheral pain receptors, and modifying individual pain perception - reducing the pain threshold and thus causing normal stimuli to be perceived as painful (3).
It is reasonable to relate BMS to such psychological disorders, since they all share common features. In effect, these disorders are all characterized by unpleasant sensations, chronic pain, and the absence of any clear organic disorder capable of accounting for the patient symptoms (3).

The classical description of depression and anxiety concomitant to BMS suggested an association between the latter and psychological problems. In addition, improvements in BMS have been observed as a result of cognitive-behavioral therapy and the use of anxiolytic drugs. This indicates that psychological disorders may predispose to the development of BMS, though the way in which they might influence its etiology remains unclear. However, some authors suggest that these psychological disorders are more a consequence of BMS than a cause of the syndrome (1).

In any case, different studies have confirmed that BMS patients have a high prevalence of psychological problems such as anxiety, depression, irritability, and an increased desire to isolate themselves, compared with the control subjects $(4,5)$.

On the other hand, although psychological factors are present in a large proportion of all cases of BMS, a substantial percentage on individuals develop the syndrome without any psychological disorders (6). This indicates that psychological disorders are not the only or exclusive cause underlying BMS.

2.- The prevalence of BMS is greater among females, and particularly in middle-aged and elderly women. The age-related reduction in estrogen and progesterone levels favors dryness of the mucosal membranes, and psychological disturbances are more frequent in middle-aged and elderly women. Based on the existing evidence, menopause can be associated to an increased risk of developing BMS (7). This hypothesis in turn is supported by the good results obtained with oral hormone (estrogen) replacement therapy in application to the symptoms of xerostomia (8).

3.- Lastly, the presence of sensory alterations such as changes in heat tolerance, reduced and altered taste sensation, and increased excitability of the blink reflex in patients with BMS points to the existence of a biological basis related to alterations of both the central and peripheral nervous system $(9,10)$. At present, BMS is regarded as a painful condition involving neuropathic mechanisms, as evidenced by the burning nature of the pain. Patients diagnosed with BMS present symptoms that are characteristic of trigeminal nerve disorders (alterations in pain perception and neuron transmission, and increased excitability of the trigeminal vascular system), suggesting the possibility that while BMS presents a multifactorial etiology, the underlying basis of the syndrome is neuropathic.

Immunohistochemical studies have shown the presence 
of alterations in the small-diameter nerve fibers $(\mathrm{C}$ fibers) in patients with BMS. However, other studies have evidenced other anatomical alterations. In effect, some patients show neuropathic signs affecting both larger- and smaller-diameter nerve fibers. It therefore may be postulated that BMS is a consequence of both generalized alterations and disorders at different levels of the trigeminal system (11).

The existence of an enhanced blink reflex in some patients with BMS points to a central nervous system alteration correlated to dopaminergic system dysfunction (9). Diminished presynaptic dopaminergic inhibition would increase dopamine release into the interneuronal space, with enhanced neuron excitability manifesting as an increased blink reflex $(9,12)$. Posteriorly, dopaminergic system dysfunction was confirmed in patients with BMS on evaluating dopaminergic function in the brainstem of such patients (13), and on observing a decrease in endogenous dopamine levels in the putamen of individuals with BMS (14).

Thus, BMS shares a range of characteristics with other forms of chronic pain (5). The alterations underlying chronic pain symptoms may result from changes in central nervous system function. Hyperstimulation of the N-methyl-D-aspartic acid (NMDA) receptors of the dorsal trunk neurons may lead to neuroplasticity phenomena secondary to hyper-excitation of these neurons (15). Neuroplasticity occurs when a neuron continuously receives nociceptive signals that produce NMDA receptor activation of the contiguous neurons - giving rise to enhanced cell sensitivity phenomena $(15,16)$. As a result of such neuron sensitization, common stimuli usually interpreted as being harmless (such as irritation produced by dentures) are reinterpreted by the central nervous system as being located above the pain threshold and therefore of a harmful nature (16). Likewise, sustained stress in denture wearers can induce continuous involuntary tongue movements-this in turn generating constant sensory stimulation capable of triggering pain sensations as a consequence of hyperstimulation, as in certain cases of BMS (10).

It has also been suggested that BMS may originate form neurological alterations located at peripheral level. Such peripheral alterations could be related to the density and/or functionality of the membrane receptors present in the oral mucosa. This hypothesis is supported by the observed local oral mucosal receptor effects of clonazepam, which to date has been found to be the most effective treatment for the symptoms of BMS (17).

Other authors have suggested that BMS behaves as a form of oral phantom pain. Some alteration in taste function would allow stimulation of the taste nerve endings to generate both excitatory and inhibitory signals (13). In healthy subjects, the chorda tympani nerve receives taste sensation from the anterior part
Table 2. Evidence supporting burning mouth syndrome as a form of oral phantom pain.

\begin{tabular}{|l|}
\hline - Bitter taste sensory capacity decreases with menopause (19). \\
\hline - Individuals with burning mouth syndrome suffer altered or abolished \\
bitter taste sensory capacity (18). The chorda tympani nerve conducts \\
information from the anterior lingual zone, where the bitter taste papillae are \\
located. Altered bitter taste sensation in patients with burning mouth \\
syndrome could reflect damage to the chorda tympani nerve. \\
\hline - In patients with burning mouth syndrome, topical anesthesia increases \\
oral burning (19,20), in the same way as topical anesthesia intensifies \\
phantom pain sensation in some patients. The increased phantom pain \\
sensations following anesthesia are explained by blockade of the inhibitory \\
activity that controls them (18,20). \\
\hline - The maximum pain intensity experienced by patients with burning mouth \\
syndrome is correlated to tongue papilla density; it therefore may be assumed \\
that patients with burning mouth syndrome who are sensitive to bitter taste \\
are super-sensitive to taste (18).
\end{tabular}

of the tongue, with inhibition of the taste response in the posterior region of the tongue, while the glossopharyngeal nerve receives taste sensation from the posterior zone of the tongue (14). It is also known that taste sensation conveyed by the chorda tympani nerve inhibits the area of the brain that receives the afferent impulses from the glossopharyngeal and trigeminal nerves. It has been suggested that damage to the chorda tympani nerve would block its inhibitory action upon the glossopharyngeal and trigeminal nerves - thus allowing intensification of the trigeminal sensations, which include oral pain, touch sensation, dry mouth and phantom sensations (18). Different findings support this theory and explain the increased prevalence of BMS in postmenopausal women (18-20) (Table 2).

In recent years, different studies have pointed to neuronal inflammation as a key element in the physiopathology of different disorders characterized by chronic pain (11). The clinical features of BMS suggest a possible participation of neuronal inflammation in the etiopathogenesis of the syndrome, along with some of the other previously considered pathogenic mechanisms. Three hypotheses have been proposed in this sense:

- A first hypothesis suggests that neuronal inflammation may be mediated by a classical axonal reflex that would activate a group of nociceptors, causing them to release neuropeptides at trigeminal terminal branch level.

- A second hypothesis points suggests the existence of an indirect reflex that would develop in the afferent nerves of the oral cavity, followed by processing at central level in the trigeminal nerve, and the induction of a response at autonomic nervous system level.

In these two models the appearance of characteristic BMS symptoms could be explained by the neurogenic 
change theory. Such change occurs when an efferent central nervous system signal produces neuropeptide release and inflammation in a zone without direct local stimulation.

- A third hypothesis is related to the capsaicin receptors, which have dual efferent and sensory activities. These receptors, which are found in the C-polymodal nociceptors, would be implicated in the regulation of the events associated to neurogenic inflammation, and would be activated upon receiving afferent signals and the simultaneous release of neuropeptides from the same nerve endings.

The neuroinflammatory model could account for the efficacy of gabaergic drugs in controlling the symptoms of BMS. These drugs would not only act at central level, since gabaergic receptor presence has also been described in peripheral tissues, and they possibly may also be present in peripheral nerve endings; as a result, the stimulation of such receptors mediated by these drugs could modulate the release of inflammatory neuropeptides. Neuroinflammation may occur in patients with normal or damaged nerve fibers, and is compatible with the histological findings reported by different authors in BMS. This hypothesis is supported by the efficacy of low-dose prazepam in controlling the symptoms of BMS in patients participating in a pilot study (11). Prazepam elicits a minimal effect at central nervous system level that is insufficient to explain resolution of the disease, and the results of the study could be interpreted in terms of the gabaergic action of the drug upon the peripheral nerve fibers (11).

\section{Discussion}

The present study has reviewed the different theories proposed to explain the etiology of primary or idiopathic burning mouth syndrome (BMS). In this context, secondary BMS should be regarded as a different process in which oral burning sensation is simply produced by some objective and tractable local or systemic condition such as hyposialia, oral infections, autoimmune disorders of the oral mucosa such as lichen planus, nutritional deficiencies, allergies, irritation attributable to esophageal reflux, the administration of certain drugs, and some endocrine disorders (21).

Precise knowledge of the etiology of BMS would allow us to define patient subpopulations with differential features, and which presently are all grouped under the term of primary BMS. A distinction has already been made among three different clinical forms of BMS which have been associated to different causes (1). The existence of non-identified subpopulations with etiologically distinct forms of BMS is fundamented upon the different therapeutic responses shown by the patients included in the different studies carried out to evaluate drug substances for the treatment of BMS. If all cases of
BMS were of one same origin, then the drugs evaluated would be expected to exhibit similar efficacy rates in all the treated patients. Another possible explanation for the differences in drug treatment response could be that the efficacy of a given agent might differ according to the stage of the disease. This would suggest the possibility of classifying BMS according to the stage of the syndrome - though no such classification has been developed to date. Each patient subpopulation would require a different management approach in order to control the symptoms and resolve the disease process. Further in-depth investigation is needed to clarify the etiopathogenic mechanisms of BMS and its triggering factors, in order to develop effective and individualized management strategies that can be extended to patients in each different primary BMS subgroup (17).

\section{Conclusion}

Although different etiological theories have been proposed to explain primary burning mouth syndrome, none have received universal acceptance to date. While the origin of BMS remains unclear, a range of factors are believed to be implicated. It seems clear that psychological factors and alterations of the central nervous system are involved, though we do not know whether they are a cause or a consequence of BMS. In addition, the influence and connections of such factors in relation to alterations at peripheral nervous system level have not been established.

\section{References References with links to Crossref-DOI}

1. Scala A, Checchi L, Montevecchi M, Marini I, Giamberardino MA. Update on burning mouth syndrome: overview and patient management. Crit Rev Oral Biol Med. 2003;14:275-91.

2. Hakeberg M, Berggren U, Hägglin C, Ahlqwist M. Reported burning mouth symptoms among middle-aged and elderly women. Eur J Oral Sci. 1997; 105:539-43

3. Low PA, Dotson RM. Symptomatic treatment of painful neuropathy. JAMA. 1998;280:1863-4

4. Bergdahl J, Anneroth G, Perris H. Personality characteristics of patients with resistant burning mouth syndrome. Acta Odontol Scand. 1995;53:7-11.

5. Grushka M. Clinical features of burning mouth syndrome. Oral Surg Oral Med Oral Pathol. 1987;63:30-6.

6. Bogetto F, Maina G, Ferro G, Carbone M, Gandolfo S. Psychiatric comorbidity in patients with burning mouth syndrome. Psychosom Med. 1998;60:378-85.

7. Wardrop RW, Hailes J, Burger H, Reade PC. Oral discomfort at menopause. Oral Surg Oral Med Oral Pathol. 1989;67:535-40.

8. Laine M, Leimola-Virtanen R. Effect of hormone replacement therapy on salivary flow rate, buffer effect and $\mathrm{pH}$ on perimenopausal and postmenopausal women. Arch Oral Biol. 1996;41:91-6.

9. Jääskeläinen SK, Forssell H, Tenovuo O. Abnormalities of the blink reflex in burning mouth syndrome. Pain. 1997;73:455-60.

10. Svensson P, Kaaber S. General health factors and denture function in patients with burning mouth syndrome and matched control subjects. J Oral Rehabil. 1995;22:887-95.

11. GuarneriF, GuarneriC, Marini H.Contribution of neuroinflammation in burning mouth syndrome: indications from benzodiazepine use. Dermatol Ther. 2008;21Suppl 2:S21-4.

12. Pajukoski H, Meurman JH, Halonen P, Sulkava R. Prevalence of 
subjective dry mouth and burning mouth in hospitalized elderly patients and outpatients in relation to saliva, medication, and systemic diseases. Oral Surg Oral Med Oral Pathol Oral Radiol Endod. 2001;92:641-9.

13. Jääskeläinen SK, Rinne JO, Forssell H, Tenovuo O, Kaasinen V, Sonninen P, et al. Role of the dopaminergic system in chronic pain -- a fluorodopa-PET study. Pain. 2001;90:257-60.

14. Hagelberg N, Forssell H, Aalto S, Rinne JO, Scheinin H, Taiminen $\mathrm{T}$, et al. Altered dopamine $\mathrm{D} 2$ receptor binding in atypical facial pain. Pain. 2003;106:43-8.

15. Woolf CJ, Thompson SW. The induction and maintenance of central sensitization is dependent on N-methyl-D-aspartic acid receptor activation; implications for the treatment of post-injury pain hypersensitivity states. Pain. 1991;44:293-9.

16. Gracely RH, Lynch SA, Bennett GJ. Painful neuropathy: altered central processing maintained dynamically by peripheral input. Pain. 1992;51:175-94.

17. Mínguez Serra MP, Salort Llorca C, Silvestre Donat FJ. Pharmacological treatment of burning mouth syndrome: A review and update. Med Oral Patol Oral Cir Bucal. 2007;12:E299-304.

18. Boras VV, Savage NW, Brailo V, Lukac J, Lukac M, Alajbeg IZ. Salivary and serum levels of substance $\mathrm{P}$, neurokinin A and calcitonin gene related peptide in burning mouth syndrome. Med Oral Patol Oral Cir Bucal. 2010;15:e427-31.

19. Bartoshuk LM. Clinical psychophysics of taste. Gerodontics. $1988 ; 4: 249-55$

20. Formaker BK, Mott AE, Frank ME. The effects of topical anesthesia on oral burning in burning mouth syndrome. Ann N Y Acad Sci. 1998;855:776-80.

21. López-Jornet P, Camacho-Alonso F, Andujar-Mateos P, SánchezSiles M, Gómez-Garcia F. Burning mouth syndrome: an update. Med Oral Patol Oral Cir Bucal. 2010;15:e562-8. 